\title{
MicroRNA-802 suppresses breast cancer proliferation through downregulation of FoxM1
}

\author{
FENG YUAN and WEI WANG \\ Department of Breast Cancer, Hubei Cancer Hospital, Wuhan, Hubei 430079, P.R. China
}

Received February 27, 2014; Accepted November 19, 2014

DOI: $10.3892 / \mathrm{mmr} .2015 .3921$

\begin{abstract}
An increasing number of studies have shown that microRNAs (miRNAs) are critical in tumor cell proliferation, as they modulate key gene transcripts. In the present study, the expression and roles of miRNA (miR)-802 were analyzed by quantitative polymerase chain reaction in breast cancer cells. The results showed that expression levels of miR-802 were significantly reduced in breast cancer tissues and cells compared with those of normal tissue and normal breast epithelial cells. In vitro and in vivo experiments demonstrated that miR-802 overexpression inhibited cell proliferation in MCF-7 breast cancer cells and tumor growth in nude mice, respectively. Furthermore, mechanistic investigation with western blotting and luciferase reporter assays revealed that miR-802 overexpression downregulated protein expression levels of Forkhead box protein M1 (FoxM1). Therefore, the results of the present study provided evidence for a previously undetermined miR-802/FoxM1 molecular network, which was involved in the regulation of breast cancer cell proliferation.
\end{abstract}

\section{Introduction}

Breast cancer has become a leading cause of mortality among females in China, with 1.1 million novel cases annually (1). Decades of studies have revealed the molecular profiling of breast cancer using expression arrays (2-4), which may aid in identifying the underlying pathogenic mechanisms and thus determining effective novel therapeutic strategies for the treatment of breast cancer.

MicroRNAs (miRNAs), a class of small endogenous RNA molecules, modulate gene expression through negatively regulating the stability or translational efficiency of specific mRNAs $(5,6)$. In addition, studies have reported the

Correspondence to: Dr Wei Wang, Department of Breast Cancer, Hubei Cancer Hospital, 116 Zhuo Dao Quan Nan Road, Wuhan, Hubei 430079, P.R. China

E-mail: weiwanghb@126.com

Key words: breast cancer, cell proliferation, microRNA, microRNA-802, Forkhead box protein M1 dysregulation of several miRNAs in breast cancer, which may contribute to tumor initiation and progression $(7,8)$. One study reported that the downregulation of miR-515-5p via the estrogen receptor, promoted breast cancer cell proliferation through modulating sphingosine kinase 1 (9), while miR-221/222 targeted adiponectin receptor 1 in order to promote epithelial-to-mesenchymal transition in breast cancer cells (10).

The function of miR-802, located on chromosome 21, in cancer biology remains to be elucidated. The present study aimed to investigate the role of miR-802 as a positive regulator of the proliferation of breast cancer cells, as well as elucidate the mechanism underlying its action in human cancers.

\section{Materials and methods}

Tissue samples. A total of 20 pairs of tumor tissues and adjacent normal tissues were obtained from patients who underwent surgery at the Department of Breast Cancer at Hubei Cancer Hospital (Wuhan, China). Written informed consent was obtained from each patient and the study was approved by the Department of Breast Cancer at Hubei Cancer Hospital institutional review board, and the Ethics Committee of Hubei Cancer Hospital.

Cell culture. Breast cancer cell lines (MCF-7, MDA-MB-453, MDA-MB-468 and ZR-75-1) and normal breast epithelial cells (HBL-100) were obtained from the Chinese Academy of Sciences Cell Bank (Shanghai, China). Cells were maintained in Dulbecco's modified Eagle's medium (Invitrogen Life Technologies, Carlsbad, CA) supplemented with $10 \%$ fetal bovine serum (Invitrogen Life Technologies). Cultures were maintained at $37^{\circ} \mathrm{C}$ in a humidified incubator containing $5 \% \mathrm{CO}_{2}$.

Quantitative polymerase chain reaction $(q P C R)$. Total RNA from tissues or cells was harvested using an miRNA Isolation kit (Ambion, Austin, TX, USA). Expression of mature miRNAs was assayed using a Taqman MicroRNA assay (Applied Biosystems, Foster City, CA, USA) specific for human miR-802. qPCR was performed using a 7900 Real-time PCR system (Applied Biosystems). All samples were normalized to the internal control, U6 small nuclear RNA. All RNA samples were examined as to their concentration and purity. RNA purity was measured using the NanoDrop ND-1000 
spectrophotometer (Thermo Fisher Scientific, Waltham, MA, USA). Based on the absorbance ratio at $260 / 280 \mathrm{~nm}$ (mean \pm standard deviation $=1.86 \pm 0.02$ ), all RNA samples were pure and protein free. The reaction conditions were as follows: Initial holding period at $95^{\circ} \mathrm{C}$ for $5 \mathrm{~min}$, then 40 cycles of $95^{\circ} \mathrm{C}$ for $5 \mathrm{sec}$ and $60^{\circ} \mathrm{C}$ for $30 \mathrm{sec}$. The primer sequences used were as follows: p27, forward 5'-ATGAGC CGCAAACTGGGTC-3' and reverse 5'-AGAGCCGAACTC CACAATCTC-3'; cyclin A, forward 5'-CGCTGGCGGTAC TGAAGTC-3' and reverse 5'-GAGGAACGGTGACATGCT CAT-3'; cyclin B1 forward 5'-AATAAGGCGAAGATCAAC ATGGC-3' and reverse 5'-TTTGTTACCAATGTCCCCAAG AG-3'. Relative quantitation analysis of gene expression data was performed according to the $2^{-\Delta \Delta \mathrm{Ct}}$ method.

Cell proliferation assay. Transfected cells were plated onto 12 -well plates at a density of $1 \times 10^{4}$ per well and cultured for 1-3 days. Cell counts were estimated by trypsinizing (Invitrogen Life Technologies) the cells and performing analysis using a Coulter counter (Beckman Coulter, Fullerton, CA, USA). For bromodeoxyuridine (BrdU; Beyotime Institute of Biotechnology, Shanghai, China) incorporation assays, a cell proliferation enzyme-linked immunosorbent assay (Beyotime Institute of Biotechnology) was used to analyze the incorporation of BrdU during DNA synthesis according to the manufacturer's instructions. Absorbance was measured at $450 \mathrm{~nm}$ using the Spectra Max 190 ELISA reader (Molecular Devices, Sunnyvale, CA, USA).

Plasmid construction and transfection. For the miR-802 expression plasmid, human miR-802 precursor was cloned by PCR and inserted into the XbaI and XhoI sites of pSilencer (Ambion). The negative control plasmid consists of a scrambled sequence (Ambion). MCF-7 cells were transfected with the miR-802 precursor or the negative control using Lipofectamine $2000^{\circledR}$ (Invitrogen Life Technologies) according to the manufacturer's instructions.

Cell cycle analysis. Cells were resuspended in $70 \%$ ethanol and fixed at room temperature for $30 \mathrm{~min}$. Cells were then washed three times with phosphate-buffered saline (PBS; Beyotime Institute of Biotechnology) and resuspended in propidium iodide (PI) stain solution, consisting of $50 \mu \mathrm{g} /$ $\mathrm{ml}$ PI (Beyotime Institute of Biotechnology) and $100 \mu \mathrm{g} / \mathrm{ml}$ ribonuclease A (Sangon Biotech, Shanghai, China) in PBS. The cells were incubated in PI stain solution for 30 min and were then analyzed by flow cytometry using a FACScalibur (BD Biosciences, Oxford, UK). The flow cytometry data were analyzed using FlowJo (Tree Star, Inc., Ashland, OR, USA).

Western blot analysis. Cells were harvested and lysed using ice-cold lysis buffer (50 mM Tris-HCl, pH 6.8; 802 mM 2-ME, $2 \% \mathrm{w} / \mathrm{v}$ SDS, and $10 \%$ glycerol). Following centrifugation at $20,000 \mathrm{x} \mathrm{g}$ for $10 \mathrm{~min}$ at $4^{\circ} \mathrm{C}$, proteins in the supernatants were quantified and separated using 10\% SDS-PAGE, then transferred to nitrocellulose membranes (GE Healthcare, Little Chalfont, UK). Following blocking with $10 \%$ non-fat milk in PBS, membranes were immunoblotted with the following primary antibodies: Anti-p27 (ab32034; rabbit monoclonal; 1:2,000), cyclin A (ab2097; rabbit polyclonal; 1:2,000),
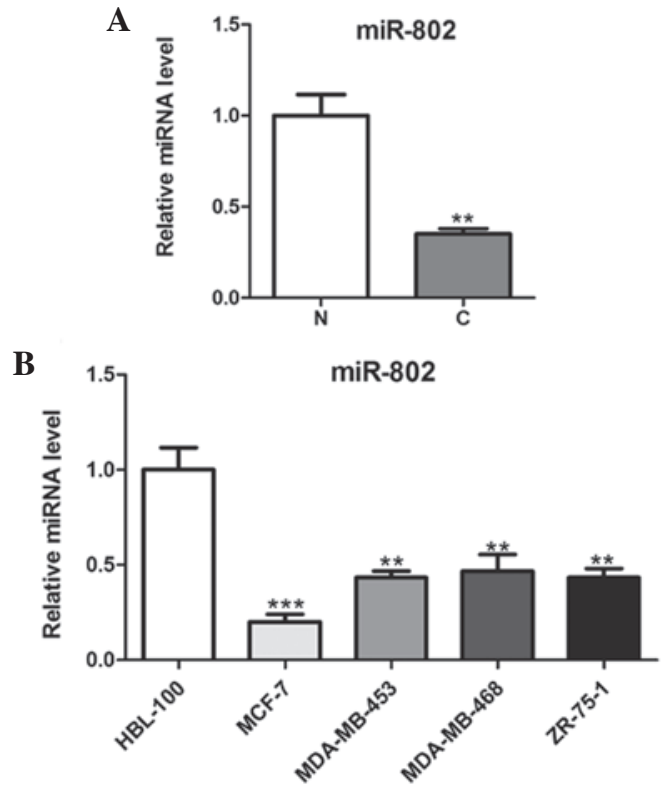

Figure 1. Expression levels of miR-802 in breast cancer tissues and cells (A) miR-802 expression was determined using TaqMan quantitative polymerase chain reaction in human breast cancer tissues and adjacent non-cancerous tissues $(\mathrm{n}=20) .{ }^{* *} \mathrm{P}<0.01$ vs. N. (B) miR-802 expression in normal breast epithelia cells (HBL-100) and breast cancer cell lines (MCF-7, MDA-MB-453, MDA-MB-468 and ZR-75-1). ${ }^{* *} \mathrm{P}<0.01$ and ${ }^{* * *} \mathrm{P}<0.001$ vs. HBL-100 cells. miR-802, microRNA-802; N, non-cancerous breast tissue; $\mathrm{C}$, breast cancer tissue.

cyclin B1 (ab72; mouse monoclonal; 1:1,000) and Forkhead box protein M1 (FoxM1) (ab83097; rabbit polyclonal; 1:1,000) antibodies purchased from Abcam (Cambridge, MA, USA). Protein levels were normalized to that of GAPDH (sc-365062; mouse monoclonal, 1:1,000; Santa Cruz Biotechnology, Inc., Dallas, TX, USA). The blots were then incubated with horseradish peroxidase-linked secondary antibodies (Cell Signaling Technologies, Inc., Danvers, MA, USA). Antibody signals were detected using a SuperSignal West Pico Chemiluminescent Substrate kit (Pierce Biotechnology, Inc., Rockford, IL, USA) according to manufacturer's instructions.

Luciferase reporter assay. The potential targets of miR-802 were analyzed by TargetScan (www.targetscan.org) and miRWalk (www.umm.uni-heidelberg.de/apps/zmf/mirwalk) softwares. Wild-type and mutant 3'-untranslated region (UTR) fragments of the FoxM1 gene were cloned into pMir-Report (Ambion), yielding pMir-Report-FoxM1. Mutations were introduced into potential miR-802 binding sites using a QuikChange site-directed mutagenesis kit (Stratagene Inc., La Jolla, CA, USA). For luciferase assays, cells were seeded in 24-well plates and transfection efficiency was normalized by cotransfecting the Simian virus 40 (SV40) plasmid. Values were determined using the Dual-Luciferase Reporter Assay system (Promega Corporation, Madison, WI, USA).

Tumor growth assay. Male BALB/c nude mice (age, five weeks) were purchased from the Experimental Animal Center of the Third Military Medical University (Chongqing, China). A total of $8 \times 10^{5}$ MCF-7 cells stably expressing miR-802 or negative control were injected subcutaneously into the skin under the 
A

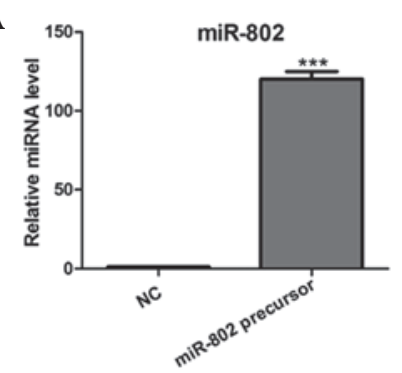

D

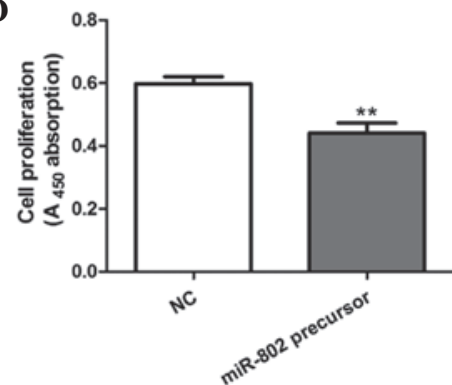

B

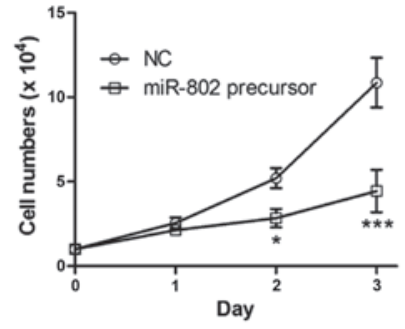

E

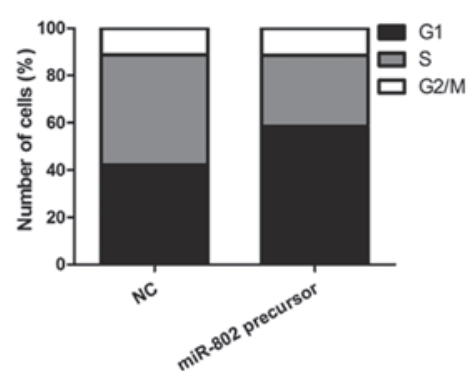

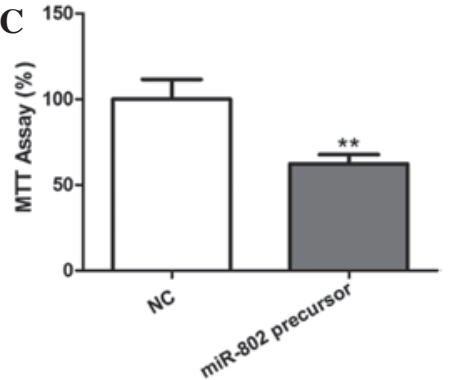

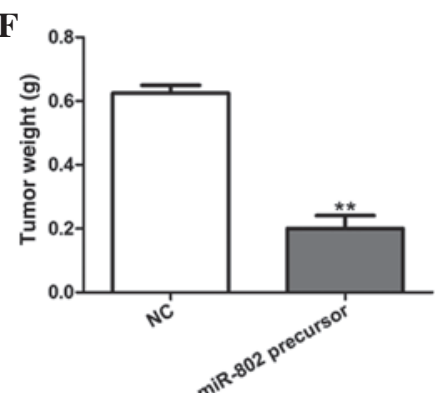

Figure 2. miR-802 overexpression inhibits breast cancer cell proliferation. MCF-7 breast cancer cells were stably transfected with an miR-802 precursor or NC for 36 h. (A) Expression of miR-802 was determined using quantitative polymerase chain reaction in MCF-7 cells following transfection. (B) Growth curve of MCF-7 cells following transfection. (C) Cell viability (MTT) and (D) proliferative potential (bromodeoxyuridine) assays were performed in MCF-7 cells following transfection. (E) Cell cycle phase analysis of transfected MCF-7 cells was performed using flow cytometry. Cells were labeled for 15 min with propidium iodide and immediately analyzed using flow cytometry. Histograms represent the percentage of cells in each phase of the cell cycle (G0/G1, $\mathrm{S}$ and G2/M). (F) Transfected MCF-7 cells were subcutaneously injected into nude mice ( $\mathrm{n}=6$ for each group) and tumor weights were measured four weeks following injection. ${ }^{* *} \mathrm{P}<0.01$ and ${ }^{* * *} \mathrm{P}<0.001$ vs. NC. miR-802, microRNA-802; NC, negative control.

A

FoxM1
5'-CUGCCAAUACGCAUCUAGGAACA-3'
miR-802

3'-CAGUAACAAAGAUUCAUCCUUGU-5'

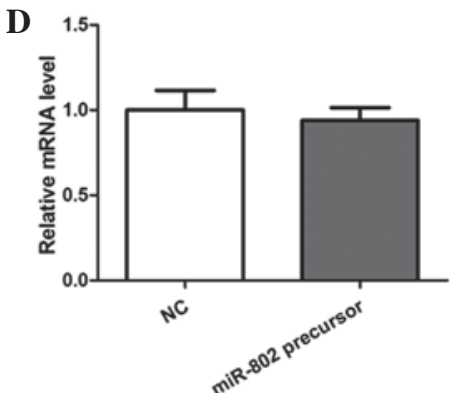

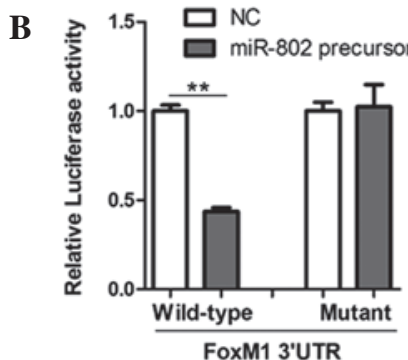

$\mathbf{E}$

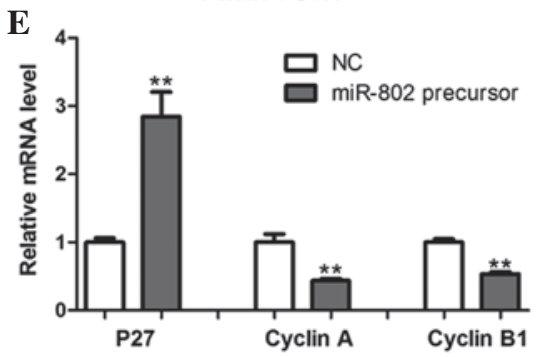

C NC miR-802 precursor

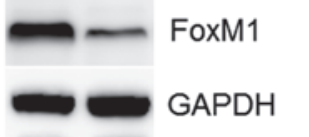

F

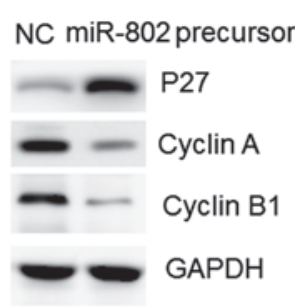

Figure 3. miR-802 represses FoxM1 expression through targeting its 3'UTR. (A) Prediction of miR-802 binding sites in the 3'UTRs of human FoxM1 gene as determined using TargetScan and miRWalk software (bold, potential binding site). (B) Luciferase reporter activity in MCF-7 breast cancer cells. Cells were transfected with $100 \mathrm{ng}$ wild-type or mutant 3'-UTR-reporter constructs together with $25 \mathrm{nM}$ miR-802 precursor or NC. (C) Protein and (D) mRNA levels of FoxM1 were determined by western blot analysis and qPCR, respectively, in MCF-7 cells transfected with miR-802 precursor or NC. (E) mRNA and (F) protein levels of p27, Cyclin A and Cyclin B1 were determined by western blot analysis and qPCR, respectively, in MCF-7 cells transfected with miR-802 precursor or NC. miR-802, microRNA-802; FoxM1, Forkhead box protein M1; UTR, untranslated region; NC, negative control; qPCR, quantitative polymerase chain reaction.

front legs of the mice. Mice were observed over four weeks for tumor formation. At four weeks, the mice were sacrificed via cervical dislocation, tumors were recovered and the wet weights of each tumor were determined. Experiments were performed using six mice per group (two groups; MCF-7 cells with stable overexpression of miR-802 precursors and negative controls).
Statistical analysis. Values are presented as the mean \pm standard error of the mean $(n>3)$. Differences between groups were analyzed using Student's t-test using GraphPad Prism software, version 6.0.1 (GraphPad Software, Inc., La Jolla, CA, USA). $\mathrm{P}<0.05, \mathrm{P}<0.01$ and $\mathrm{P}<0.001$ were considered to indicate statistically significant differences. 

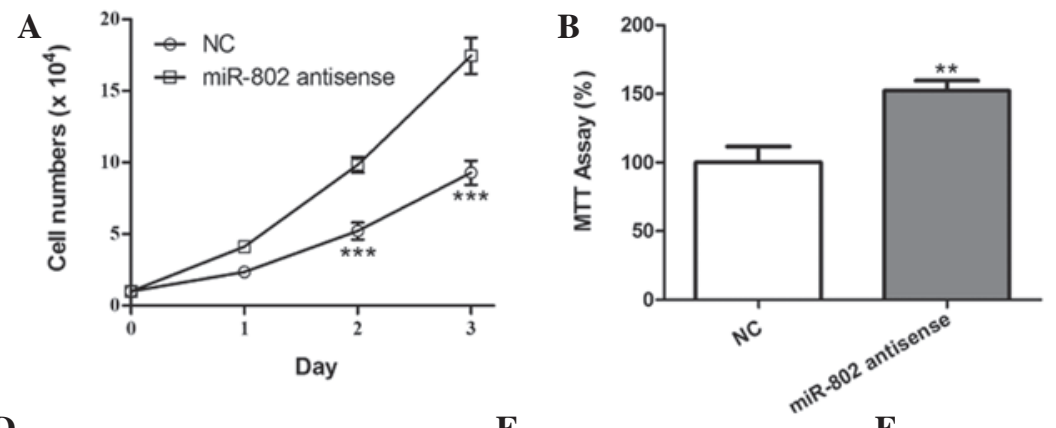

$\mathbf{E}$

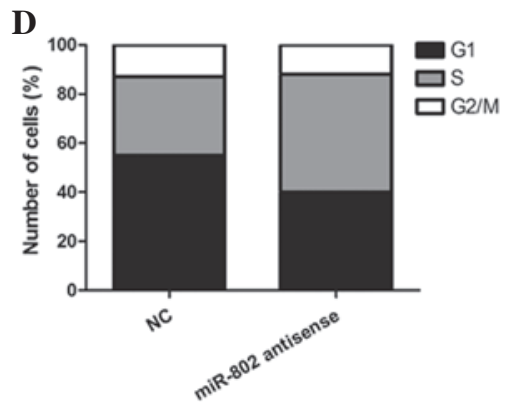

NC miR-802 antisense

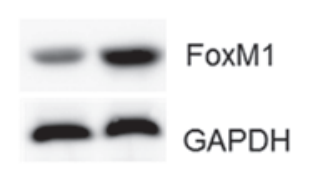

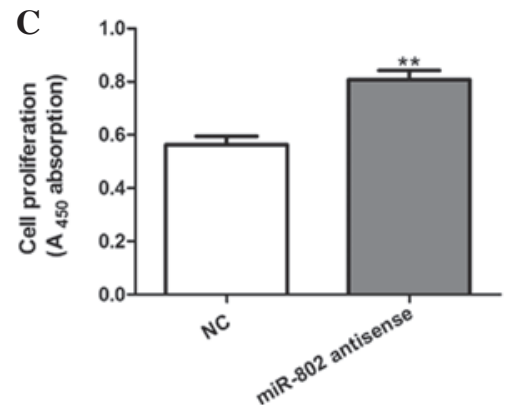

G
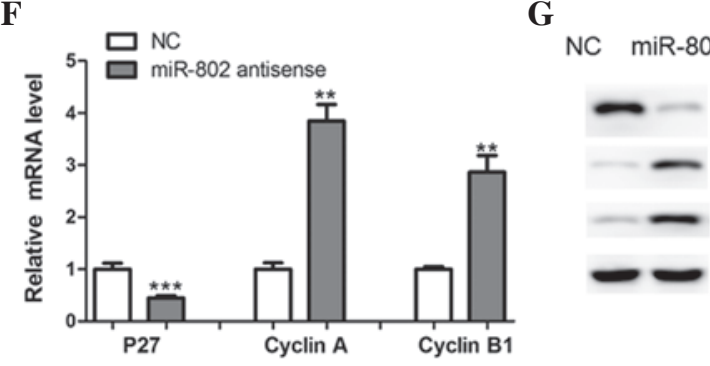

P27

Cyclin A

Cyclin B1

GAPDH

Figure 4. miR-802 antisense promotes the proliferation of breast cancer cells. MCF-7 breast cancer cells were transfected with miR-802 antisense or NC. (A) Growth curve of MCF-7 cells following transfection. (B) Cell viability (MTT) and (C) proliferative potential (bromodeoxyuridine) assays were performed in MCF-7 cells following transfection. (D) Cell cycle phase analysis of transfected MCF-7 cells was performed using flow cytometry. (E) Western blot analysis of FoxM1 in transfected MCF-7 cells. (F) mRNA and (G) protein levels of p27, Cyclin A and Cyclin B1 were determined using western blot analysis and quantitative polymerase chain reaction, respectively, in MCF-7 cells following transfection. miR-802, microRNA-802; FoxM1, Forkhead box protein M1; NC, negative control.

\section{Results}

miR-802 is downregulated in breast cancer tissues and cells. In order to explore the role of miR-802 in breast cancer carcinogenesis, microRNAs were extracted from malignant and normal breast tissue biopsies and then analyzed using qPCR. As shown in Fig. 1A, miR-802 expression was significantly downregulated in cancer tissues compared with that of the adjacent non-cancerous tissues. In addition, miR-802 expression was significantly decreased in the breast cancer cell lines compared with that of the normal breast epithelial cells (Fig. 1B).

miR-802 overexpression inhibits breast cancer cell proliferation in vitro and in vivo. In order to determine the effect of miR-802 on breast cancer cell growth, MCF-7 cells overexpressing miR-802 precursor were constructed, which led to a significant increase in miR-802 expression (Fig. 2A). As a result, miR-802 overexpression significantly inhibited the proliferative ability of MCF-7 cells post-transfection compared with that of the negative control (Fig. 2B-D). Furthermore, cell cycle analysis revealed that a significantly higher percentage of cells overexpressing miR-802 were in G1/G0 phase and a decreased percentage of these cells were in $S$ phase, compared with that of the negative control-transfected cells (Fig. 2E).

MCF-7 cells with stable overexpression of miR-802 were then evaluated for tumorigenic potential in vivo. Cells were injected subcutaneously into the skin under the front legs of nude mice and tumor growth was closely monitored for four weeks. As a result, the tumor size and weight was markedly reduced in miR-802-overexpressing tumors compared with that of the control tumors (Fig. 2F), suggesting that miR-802 suppressed breast cancer growth in vivo.
miR-802 targets the FoxM1 3'UTR and downregulates its expression. In order to understand the underlying mechanisms of miR-802-induced growth inhibition in MCF-7 cells, potential targets of miR-802 were searched using TargetScan and miRWalk software. The results showed that FoxM1, an oncogene in human cancer, harbored a potential miR-802 binding site (Fig. 3A). In order to verify whether FoxM1 is a direct target of miR-802, a luciferase reporter vector was constructed, which contained the putative miR-802 binding sites within the FoxM1 3'-UTR. As shown in Fig. 3B, miR-802 overexpression significantly repressed luciferase activity when the reporter construct contained the FoxM1 3'UTR in MCF-7 cells (Fig. 3B). However, mutation of the miR-802 binding site from the FoxM1 3'-UTR abolished the effect of miR-802, suggesting that miR-802 directly inhibited FoxM1 expression through targeting its 3'-UTR (Fig. 3B).

A shown in Fig. 3C, western blot analysis revealed that the miR-802 precursor significantly decreased the protein expression of FoxM1, while its mRNA levels remained unchanged (Fig. 3D). Therefore, the results suggested that miR-802 negatively regulated FoxM1 expression at the translational level.

FoxM1 was previously reported to transcriptionally downregulate the expression of p27, while upregulating Cyclin A and Cyclin B1, key regulators of cell-cycle progression (11). In concurrence with this previous study, the present study observed the enhanced expression of p27 and downregulation of Cyclin A and Cyclin B1 in MCF-7 cells overexpressing miR-802 (Fig. 3E and F). Therefore, these results further indicated that FoxM1 was an important target gene of miR-802 in breast cancer cells.

miR-802 antisense promotes the proliferation of breast cancer cells. MCF-7 cells were transfected with miR-802 antisense 
in order to block the functions of endogenous miR-802. As a result, ectopic expression of the miR-802 antisense led to the increased proliferative ability of MCF-7 cells, compared with that of the negative control-transfected cells (Fig. 4A-C). In addition, the inhibition of miR-802 significantly reduced the percentage of cells in G0/G1 phase and increased the percentage of cells in S phase (Fig. 4D). Furthermore, protein levels of FoxM1 were upregulated in MCF-7 cells transfected with miR-802 antisense (Fig. 4E). The downregulation of p27 and upregulation of Cyclin A and Cyclin B1 was also observed following miR-802 antisense transfection (Fig. 4F and G). These results therefore supported the conclusion that miR-802 regulated FoxM1 expression in breast cancer cells.

\section{Discussion}

miR-802 has been shown to modulate the biological efficacy of Ang II in the human gastrointestinal tract via downregulation of angiotensin II type 1 receptor (12). In addition, the role of miR-802 in the development of obesity-associated impairment of hepatic glucose metabolism was previously identified (13). In the present study, miR-802 expression and its role in breast cancer were determined and suggested its potential therapeutic tumor suppressor role in breast cancer. Cell viability assays and cell cycle analysis demonstrated that selective overexpression of miR-802 inhibited the proliferative ability of MCF-7 breast cancer cells, while inhibition of miR-802 promoted cell proliferation. In addition, FoxM1 was identified as a novel direct target of miR-802 using a luciferase reporter assay and western blot analysis in MCF-7 cells. However, larger sample sizes are required in order to further verify the results of the present study. In addition, further studies are also required in order to determine whether miR-802 may be used as a biomarker for the diagnosis and prognosis in breast cancer. Furthermore, it may be of interest to further investigate whether miR-802 is dysregulated in other types of human cancers.

FoxM1 is a member of the Forkhead transcription factor family, which control cell proliferation and apoptosis through the regulation of genes associated with cell cycle entry, including p21 and p27 (14,15). Previous studies have reported that FoxM1 was upregulated in breast cancer and its expression was correlated with poor prognosis and metastasis in breast cancer $(16,17)$. Therefore, FoxM1 has become a target for therapeutic intervention in cancer treatment. The combination of oxidative stress and FoxM1 inhibitors has been reported to induce apoptosis in cancer cells as well as inhibit xenograft tumor growth (18). However, the molecular determinants for the upregulation of FoxM1 remain to be elucidated. Previous studies suggested that FoxM1 may be regulated by several miRNAs, including miR-134, miR-149 and miR-370 (19-21). This therefore indicated that the dysregulated expression of certain miRNAs may have a critical role in the aberrant expression of FoxM1 in human cancers.

In conclusion, the results of the present study provided the first evidence that overexpression of miR-802 inhibited breast cancer cell growth in vitro and in vivo through regulating
FoxM1 expression. This therefore indicated the important role of miR-802 in breast cancer pathogenesis and implicated its potential therapeutic use for the treatment of breast cancer.

\section{References}

1. Cao W, Wang X and Li JC: Hereditary breast cancer in the Han Chinese population. J Epidemiol 23: 75-84, 2013.

2. Assadi M, Lamerz J, Jarutat T, et al: Multiple protein analysis of formalin-fixed and paraffin-embedded tissue samples with reverse phase protein arrays. Mol Cell Proteomics 12: 2615-2622, 2013.

3. Asghar U, Witkiewicz AK, Turner NC and Knudsen ES: The history and future of targeting cyclin-dependent kinases in cancer therapy. Nat Rev Drug Discov 14: 130-146, 2015.

4. Atalay C: New concepts in axillary management of breast cancer. World J Clin Oncol 5: 895-900, 2014.

5. Van Kouwenhove M, Kedde M and Agami R: MicroRNA regulation by RNA-binding proteins and its implications for cancer. Nat Rev Cancer 11: 644-656, 2011.

6. Ameres SL and Zamore PD: Diversifying microRNA sequence and function. Nat Rev Mol Cell Biol 14: 475-488, 2013.

7. Singh R and Mo YY: Role of microRNAs in breast cancer. Cancer Biol Ther 14: 201-212, 2013.

8. Redova M, Sana $\mathbf{J}$ and Slaby O: Circulating miRNAs as new blood-based biomarkers for solid cancers. Future Oncol 9: 387-402, 2013.

9. Pinho FG, Frampton AE, Nunes J, et al: Downregulation of microRNA-515-5p by the estrogen receptor modulates sphingosine kinase 1 and breast cancer cell proliferation. Cancer Res 73: 5936-5948, 2013.

10. Hwang MS, Yu N, Stinson SY, et al: miR-221/222 targets adiponectin receptor 1 to promote the epithelial-to-mesenchymal transition in breast cancer. PloS One 8: e66502, 2013.

11. Wang IC, Chen YJ, Hughes D, et al: Forkhead box M1 regulates the transcriptional network of genes essential for mitotic progression and genes encoding the SCF (Skp2-Cks1) ubiquitin ligase. Mol Cell Biol 25: 10875-10894, 2005.

12. Sansom SE, Nuovo GJ, Martin MM, Kotha SR, Parinandi NL and Elton TS: miR-802 regulates human angiotensin II type 1 receptor expression in intestinal epithelial C2BBel cells. Am J Physiol Gastrointest Liver Physiol 299: G632-G642, 2010.

13. Kornfeld JW, Baitzel C, Konner AC, et al: Obesity-induced overexpression of miR-802 impairs glucose metabolism through silencing of Hnf1b. Nature 494: 111-115, 2013.

14. Sengupta A, Kalinichenko VV and Yutzey KE: FoxO1 and FoxM1 transcription factors have antagonistic functions in neonatal cardiomyocyte cell-cycle withdrawal and IGF1 gene regulation. Circ Res 112: 267-277, 2013.

15. Qu K, Xu X, Liu C, et al: Negative regulation of transcription factor FoxM1 by p53 enhances oxaliplatin-induced senescence in hepatocellular carcinoma. Cancer Lett 331: 105-114, 2013.

16. Sanders DA, Ross-Innes CS, Beraldi D, Carroll JS and Balasubramanian S: Genome-wide mapping of FOXM1 binding reveals co-binding with estrogen receptor alpha in breast cancer cells. Genome Biol 14: R6, 2013.

17. Yang $\mathrm{C}$, Chen $\mathrm{H}$, Tan $\mathrm{G}$, et al: FOXM1 promotes the epithelial to mesenchymal transition by stimulating the transcription of Slug in human breast cancer. Cancer Lett 340: 104-112, 2013.

18. Halasi M, Pandit B, Wang M, Nogueira V, Hay N and Gartel AL: Combination of oxidative stress and FOXM1 inhibitors induces apoptosis in cancer cells and inhibits xenograft tumor growth. Am J Pathol 183: 257-265, 2013.

19. Li J, Wang Y, Luo J, et al: miR-134 inhibits epithelial to mesenchymal transition by targeting FOXM1 in non-small cell lung cancer cells. FEBS Lett 586: 3761-3765, 2012.

20. Ke Y, Zhao W, Xiong J and Cao R: miR-149 Inhibits non-small-cell lung cancer cells EMT by targeting FOXM1. Biochem Res Int 2013: 506731, 2013.

21. Feng Y, Wang L, Zeng J, et al: FoxM1 is overexpressed in Helicobacter pylori-induced gastric carcinogenesis and is negatively regulated by miR-370. Mol Cancer Res 11: 834-844, 2013. 